\title{
High-resolution X-ray spectra of quasars
}

\author{
Shai Kaspi ${ }^{1,2}$ \\ ${ }^{1}$ School of Physics \& Astronomy and the Wise Observatory, The Raymond and Beverly Sackler \\ Faculty of Exact Sciences, Tel-Aviv University, Tel-Aviv 69978, Israel email: shai@wise.tau.ac.il \\ ${ }^{2}$ Physics Department, Technion, Haifa 32000, Israel
}

\begin{abstract}
Past X-ray observations by $A S C A$ suggest that warm absorbers (O vII and O viII edges) are apparently rare in high luminosity AGNs (quasars) while they are more common in low luminosity AGNs (Seyferts). However, this could be a selection effect if high luminosity AGNs have mostly narrow absorption lines (with no strong bound free edges), which escaped detection by the low resolution of $A S C A$. To check this hypothesis we are studying the highresolution X-ray spectra of quasars from grating spectrometers on board Chandra and XMMNewton in search for absorption lines. In this contribution we present spectra of three quasars. The spectra show narrow (several hundred $\mathrm{km} \mathrm{s}^{-1}$ ) absorption and emission X-ray lines from $\mathrm{H}$-like and He-like ions of $\mathrm{O}, \mathrm{Ne}, \mathrm{Mg}$, and other abundant elements. We also detect absorption from iron L-shell lines and iron M-shell unresolved transition array. We present the analysis of MR2251-178 where we find that at least two, and probably three, distinct warm absorbers are needed to explain the high resolution spectrum of this object. We re-analyze the highresolution X-ray spectrum of PG $1211+143$ and suggest that an outflow velocity of $\sim 3000$ $\mathrm{km} \mathrm{s}^{-1}$ provides an adequate explanation to these data. We also present preliminary results form the Chandra/HETGS observation of the quasar 4C74.26.
\end{abstract}

\section{Introduction}

A significant fraction (50-75\%) of Seyfert-I Active Galactic Nuclei (AGNs) exhibit absorption features due to highly ionized gas (so called "warm absorber" - WA) along the line-of-sight to the central source (e.g., George et al. 1998). Early $A S C A$ spectra showed only the strongest bound-free O VII and O VIII edges, while the full complexity of the absorption spectrum are clearly evident in recent Chandra and XMM-Newton observations. The X-ray grating spectrometers aboard these two observatories reveal narrow absorption lines from $\mathrm{H}$-like and He-like ions as well as from lower ionization ions of all abundant elements from carbon to iron, and M-shell and L-shell absorption lines from iron ions. These narrow absorption lines were found in most of Seyfert I AGNs observed so far (e.g., NGC 5548 - Steenbrugge et al. 2003; NGC 4051 - Collinge et al. 2001). In most cases the absorption lines are blueshifted by about several hundred $\mathrm{km} \mathrm{s}^{-1}$ relative to the systemic velocity, indicating that the ionized absorbing gas is outflowing.

The best example, so far, is the Seyfert-I galaxy NGC 3783, which has the best observed X-ray spectrum in terms of spectral resolution and S/N. It was observed for $900 \mathrm{ks}$ with the High Energy Transmission Grating Spectrometer (HETGS) on Chandra (Kaspi et al. 2002; Netzer et al. 2003) and for about $280 \mathrm{ks}$ with the Reflection Grating Spectrometer (RGS) on XMM-Newton (Behar et al. 2003). The spectrum shows more than 150 absorption lines from more than 30 ions spanning a wide range of ionization potentials. In order to model this spectrum Netzer et al. (2003) invoked a photoionization model which includes three ionized components, each split into two kinematic components (at outflow velocities of 500 and $1000 \mathrm{~km} \mathrm{~s}^{-1}$ ). The three components span a large range of ionization and have total column density of about $4 \times 10^{22} \mathrm{~cm}^{-2}$. All three components are thermally stable and seem to have the same gas pressure. Due to the detailed study 
of this spectrum it can serve as a benchmark for the study of other high resolution X-ray spectra of AGNs.

In contrast to the many studies of the high-resolution X-ray spectra of low-luminosity AGNs, there were only few such studies of high-luminosity AGNs - the quasars. Studies by $A S C A$ (e.g., George et al. 2000) show that the fraction of these with strong WAs is below the equivalent fraction in low-luminosity AGNs. A plausible explanation can be that the WA in high-luminosity AGNs is much more ionized and thus escaped the detection by the low resolution of $A S C A$, which is only sensitive to the strong bound-free absorption by the O VII and O VIII edges. To investigate this hypothesis we are studying the X-ray high resolution grating spectra of quasars from Chandra and XMM-Newton in search for absorption lines. In this contribution we present some preliminary results for three of the quasars we are studying.

\section{MR 2251+178}

$\operatorname{MR} 2251+178(z=0.6398 \pm 0.00006, V \approx 14)$ is the first quasar detected by X-ray observations. In Figure 1 we present the high-resolution grating spectrum obtained by the RGS on May 2002. The spectrum shows emission lines from N vi, O viI, O viII, Ne IX, and Nex, as well as absorption lines from O III, O IV, O v, O vi, Ne IX and Ne X. Several other absorption lines which we suspect their presence are marked in Figure 1.

In order to fit this high-resolution spectrum we first fitted the low resolution EPIC-pn data obtained in the same observation. The EPIC-pn data clearly show a power law with a photon index of $\Gamma \approx 1.6$ at energies above $3 \mathrm{keV}$. Extrapolating this power law to lower energies revels the presence of a WA around $0.8 \mathrm{keV}$, an additional absorber below $0.5 \mathrm{keV}$, and some excess emission around $0.5 \mathrm{keV}$. Our best fitted model for these data yields a WA with a column density, $N_{\mathrm{H}}$, of $10^{21.51 \pm 0.03} \mathrm{~cm}^{-2}$, ionization parameter, $U_{\text {OX }}$, of $10^{-1.78 \pm 0.05}$ and a line of sight covering factor of 0.8 . Assuming gas with the same properties produces the emission, we find a global covering factor of 0.3 . For the less ionized absorber we find that it can be fitted with a neutral absorber (in addition to the galactic one) with $N_{\mathrm{H}} \approx 10^{20.3} \mathrm{~cm}^{-2}$. This absorber can also be modeled as a combination of low-ionization absorber with $U_{\mathrm{OX}} \approx 10^{-4}$ and $N_{\mathrm{H}} \approx 10^{20.3} \mathrm{~cm}^{-2}$ and a neutral absorber of $N_{\mathrm{H}} \approx 10^{20.06} \mathrm{~cm}^{-2}$. Both cases give equally good fits.

Modeling of the RGS spectrum was done in two steps. First we experimented with a two-components absorber similar to the one fitted to the EPIC-pn data described above, which involves a highly-ionized absorber and a second absorber of much lower-ionization. We experimented with a range of parameters around the values found for the EPIC-pn. The parameters we found to fit best for the two absorbers in the RGS spectrum are: $\log \left(U_{\mathrm{OX}}\right)=-1.68$ and $N_{\mathrm{H}}=10^{21.8} \mathrm{~cm}^{-2}$ for the high-ionization WA component and $\log \left(U_{\mathrm{OX}}\right)=-4.0$ and $N_{\mathrm{H}}=10^{20.3} \mathrm{~cm}^{-2}$ for the low-ionization component. The second step includes a three-components absorber. The main motivation for this is the fact that the RGS data around 16-17 $\AA$ clearly falls below the two-components model. The excess absorption is probably caused by the unresolved transition array (UTA) of iron M-shell lines which has been observed in several other AGNs (see, e.g., Netzer et al. 2003 for the case of NGC 3783). Our photoionization code includes all these lines but the two-components WA produces too shallow a feature at too short a wavelength. We find that an additional shell with a column density of $10^{21.3} \mathrm{~cm}^{-2}$ and $\log \left(U_{\mathrm{OX}}\right)=-2.6$ can significantly improve the fit. This component produces a noticeable UTA feature and contributes also to the observed O VII emission. This requires lowering the emission from the high-ionization component by about $20 \%$ to produce an adequate fit to all emission lines. Adding this component force us to increase the ionization parameter of the highly 


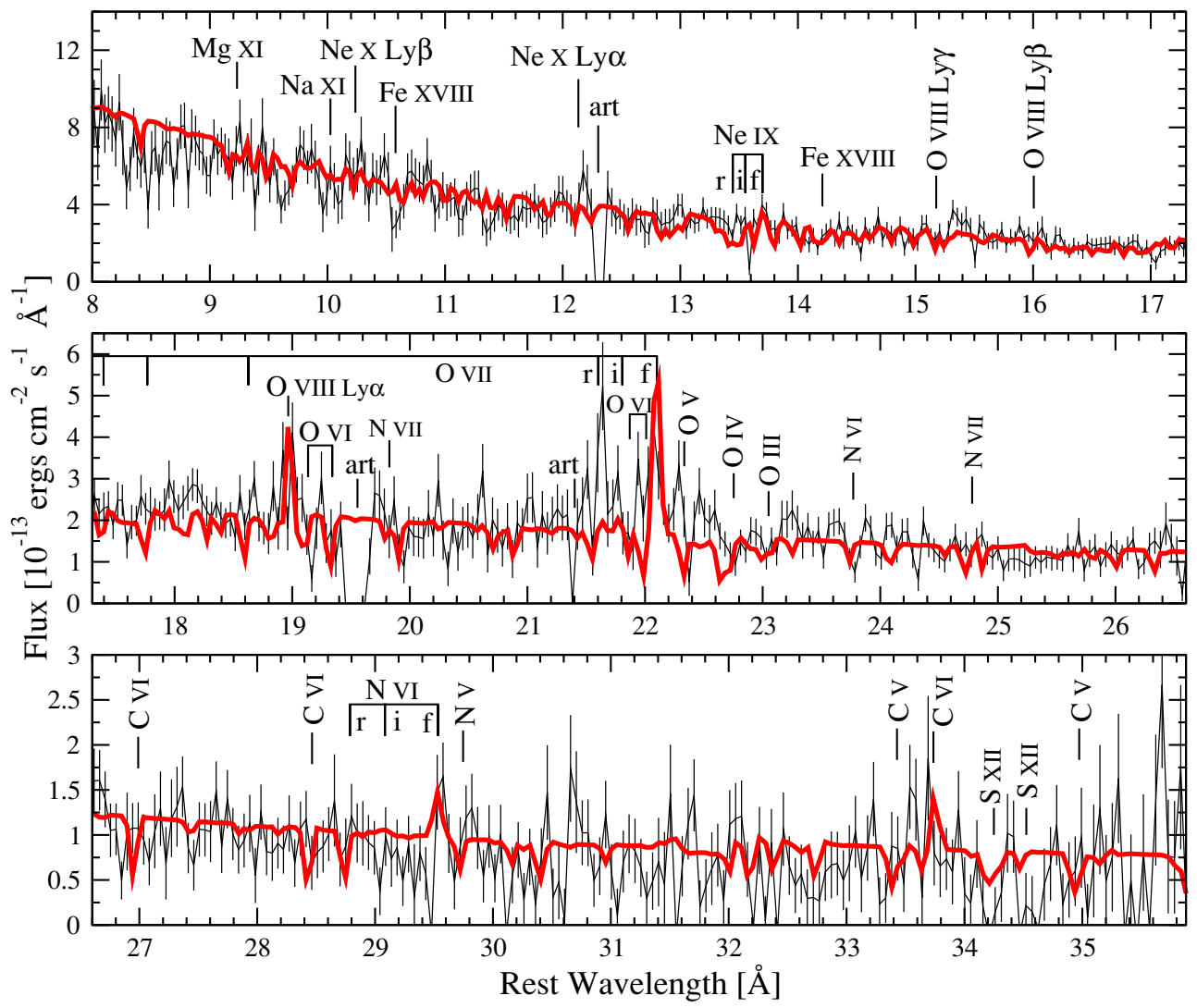

Figure 1. Combined RGS1 and RGS2 spectrum of MR $2251+178$ binned to $\sim 0.04 \AA$. The spectrum has been corrected for Galactic absorption. The strongest emission lines are due to the $\mathrm{O}$ vil triplet and $\mathrm{O}$ VIII Ly $\alpha$. Other suggested emission and absorption lines are marked. Gaps in the spectrum due to chip gaps are marked as 'art'. The three absorbers model was convolved with the RGS instrumental resolution and was also binned to $0.04 \AA$.

ionized gas (the one with column density of $10^{21.8} \mathrm{~cm}^{-2}$ ) to $\log \left(U_{\mathrm{OX}}\right)=-1.4$. We note that the mean $U_{\text {OX }}$ of these two WA components is the same as the one found earlier in the two-components model. The three-components model is compared with the RGS data in Figure 1.

\section{3. $\mathrm{PG} 1211+143$}

PG $1211+143(z=0.0809 \pm 0.0005, V \approx 14.5)$ is a radio-quiet narrow emission line quasar. It was observed with XMM-Newton for about $60 \mathrm{ks}$ on June 2001. Using the EPIC-pn, EPIC-MOS, and RGS data Pounds et al. (2003) identified absorption lines from H-like and He-like ions of Fe, S, Mg, Ne, O, N, and C. They claimed the observed line energies indicate an ionized outflow velocity of $\sim 24000 \mathrm{~km} \mathrm{~s}^{-1}$. We obtained the data from the $X M M-N e w t o n$ archive and present in Figure 2 the RGS data. Our preliminary re-analysis of the data shows that an ionized absorber with outflow velocity of about $3000 \mathrm{~km} \mathrm{~s}^{-1}$ provides an alternative explanation to the data. We are able to identify at this velocity a series of absorption lines from $\mathrm{H}$-like and He-like ions of $\mathrm{Mg}, \mathrm{Ne}$, and $\mathrm{O}$, as well as L-shell lines from iron ions. We also include the emission seen from the O vII and Ne Ix forbidden lines, and the Ly $\alpha$ emission lines of O VIII and Ne X. These emission 


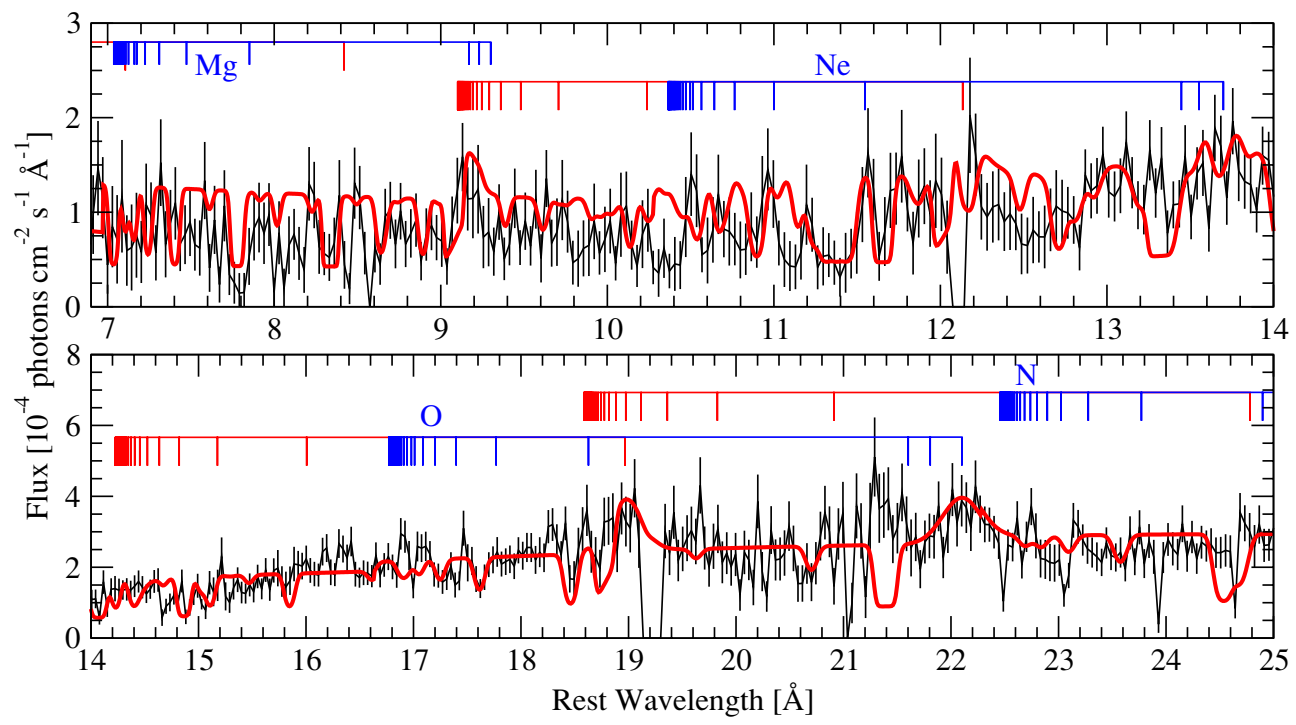

Figure 2. Combined RGS1 and RGS2 spectrum of PG $1211+143$ binned to $\sim 0.04 \AA$. The spectrum has been corrected for Galactic absorption. The model shown includes absorption lines blueshifted by $3000 \mathrm{~km} \mathrm{~s}^{-1}$ and few emission lines. Theoretical rest-frame wavelengths of $\mathrm{H}$-like and He-like ions of $\mathrm{N}, \mathrm{O}, \mathrm{Ne}$, and $\mathrm{Mg}$ are marked above the spectrum.

lines together with the many L-shell iron lines were not taken into account in the model by Pounds et al. (2003).

\section{4. $4 \mathrm{C} 74.26$}

$4 \mathrm{C} 74.26(z=0.104, V \approx 14.8)$ is a radio loud quasar with relatively strong X-ray flux compared with other quasars. It was observed with Chandra/HETGS on December 2003 for $70 \mathrm{ks}$. A preliminary analysis of the data revels emission and absorption lines from $\mathrm{H}$-like and $\mathrm{He}$-like ions of $\mathrm{Mg}, \mathrm{Al}$, and $\mathrm{Si}$, as well as lower ionization Si absorption lines. These data, as well as the ones presented in the previous sections, indicate that highly-ionized WAs are present in quasars and can be detected with the power of high resolution X-ray spectroscopy.

\section{Acknowledgements}

I am grateful to my collaborators in the studies presented here, Hagai Netzer, Ehud Behar, Doron Chelouche, Ian, M. George, Kirpal Nandra, and T.J. Turner. I acknowledge a financial support by the Israel Science Foundation grant no. 545/00.

\section{References}

Behar, E., et al. 2003, ApJ, 598, 232

Collinge, M. J., et al. 2001, ApJ, 557, 2

George, I. M., et al. 1998, ApJS, 114, 73

George, I. M., et al. 2000, ApJ, 531, 52

Kaspi, S., et al. 2002, ApJ, 574, 643

Netzer, H., et al. 2003, ApJ, 599, 933

Pounds, K. A., et al. 2003, MNRAS, 345, 705

Steenbrugge, K. C., Kaastra, J. S., de Vries, C. P., \& Edelson, R. 2003, A\&A, 402, 477 